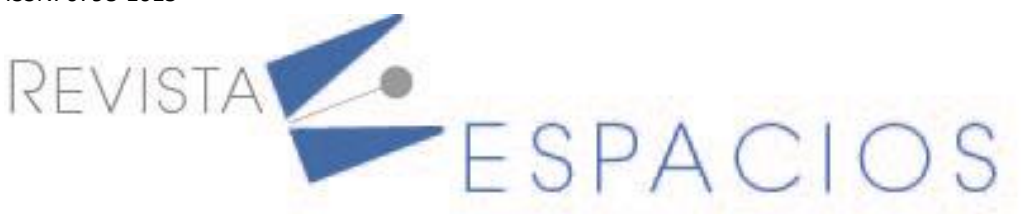

\title{
Retorno de la inversión social de la estrategia de internacionalización de la MiPymes agrícolas
}

\author{
Evaluation of Strategic Planning considering corporate social responsibility
}

\author{
BRIONES, Víctor H. ${ }^{1}$ \\ MENDOZA, Henry ${ }^{2}$ \\ SEGARRA, Pedro ${ }^{3}$ \\ VILLEGAS, María F. ${ }^{4}$
}

\section{Resumen}

La responsabilidad social corporativa (RSC) es ampliamente detallada en los medios de investigación. La planeación estratégica cumple un tratamiento similar. La presente investigación, tiene como objetivos relacionar ambas variables provenientes de las ciencias administrativas, identificar la función de ambas variables y proponer la forma de que se evalúe su relación. Esta relación se la investiga en la racionalización y la coherente participación de la empresa en la sociedad evocando la innovación en la misión empresarial.

Palabras clave: planeación estratégica, responsabilidad social corporativa, retorno de la inversión social.

\begin{abstract}
Corporate social responsibility is extensively detailed in the research media. Strategic planning follows a similar treatment. The objective of this research is to relate both variables from the administrative sciences, identify the function of both variables and propose how their relationship is evaluated. This relationship is investigated in the rationalization and coherent participation of the company in society, evoking innovation in the business mission.

key words: strategic planning, corporate social responsibility, return on social investment.
\end{abstract}

\section{Introducción}

Un grupo de investigadores de la Universidad de Guayaquil, buscó la forma de identificar las estrategias empresariales sustentables de las MiPymes de frutas y hortalizas no tradicionales de las zonas de desarrollo $5 \mathrm{y}$ 8 del Ecuador y encontró que la responsabilidad social corporativa podría adaptarse perfectamente para comercializar sus productos a mercados europeos que dan preferencias comerciales a empresas socialmente responsables. Para poder determinar los efectos de las estrategias empresariales sustentables en el proceso de internacionalización se deberá tener un modelo de valoración (retorno de inversión) que relacione la estrategia

\footnotetext{
${ }^{1}$ Docente titular. Facultad de Ciencias Administrativas. Universidad de Guayaquil. victor.brionesk@ug.edu.ec

${ }^{2}$ Docente titular. Facultad de Ciencias Administrativas. Universidad de Guayaquil. henry.mendozaavi@ug.edu.ec

${ }^{3}$ Investigador independiente. Universidad de Guayaquil. humberto.segarraj@ug.edu.ec

${ }^{4}$ Investigador independiente. Universidad de Guayaquil. maria.villegasva@ug.edu.ec
} 
y la responsabilidad social corporativa en los resultados que se informan a los observatorios que acrediten la misma a los compradores de frutas y hortalizas en Europa.

Esta relación se la investiga en la racionalización y la coherente participación de la empresa en la sociedad evocando la innovación en la misión empresarial, así como su visión y orientación, luego de repensar a la organización en tiempos de crisis por factores que están fuera del control del gerente, tales como, desastres naturales, sanitarios, sociales y existenciales (Flores \& Cano, 2016). El resultado es la formulación dialéctica de un modelo de normativa participativa y descentralizada, es decir de la planeación compartida de la empresa y la sociedad (Moura-Leite \& Padgett, 2014). La estrategia sirve como base para la creación de una empresa comercial, al tiempo que establece su posición en el mercado, su competitividad y su existencia continua (Mary Saltos \& Muñoz Moreta, 2019). Para lograr estos aspectos, se requiere planificación / programación para elaborar o formular y renovar / cambiar la estrategia según lo requieran las empresas (Miguel Saltos \& Velazquez, 2019). Esta planificación o programación se lleva a cabo en gran medida dentro del contexto de las dimensiones estratégicas que se describirán posteriormente. Por lo tanto, en esta sección, la RSC se explora dentro de cada dimensión. Sin embargo, esta exploración no está impulsada por el proceso. Es decir, el interés radica en cómo la RSC podría integrarse más plenamente en la estrategia de la empresa, y en qué pasos debe tomar una empresa para formular la estrategia(Robin \& Reidenbach, 1988). En este sentido, la discusión no es secuencial, sino que examina los componentes constitutivos de la estrategia y cómo la RSC podría integrarse en cada componente.

Se esperaría que este enfoque conduzca a una integración más estrecha de la RSC con la estrategia, pero el propósito es plantear el camino correcto a seguir o el proceso a seguir. De hecho, la integración de la RSC más plenamente con la estrategia es probable que sea un patrón emergente a lo largo del tiempo, en lugar de una ruta prescrita y con un límite de tiempo, pero es mejor saber dónde empezar y hasta dónde llegar. Aunque los programas de RSC involucran una multitud de actividades actuales, uno puede notar que su implementación comienza con el proceso de planificación estratégica (Masud et al., 2019). El proceso de planificación tiene como fin fijar los objetivos, estrategias que apuntan a lograrlos y establecer el calendario para la asignación de recursos financieros, materiales y humanos (Sousa et al., 2010). La Responsabilidad Social Corporativa (RSC) no es un concepto nuevo para las empresas, pero se está convirtiendo rápidamente en un componente fundamental para el éxito. Para muchas empresas, la razón principal para ser socialmente responsable es bastante simple; Es lo correcto. Originalmente, este sentimiento fue el factor impulsor de la comunidad y el compromiso social de una empresa. Sin embargo, en los últimos años, los consumidores y los empleados han presionado a las empresas para que se comprometan con sus comunidades y la sociedad en general. El resultado es ganar / ganar para las comunidades locales y las organizaciones de caridad, así como para las empresas (Moore, 2020).

Los académicos han prestado considerable atención al estudio de la relación entre la RSC y el desempeño de la empresa. Sin embargo, poca investigación empírica demuestra lo que realmente forma o impulsa la RSC. Este documento desarrolla el caso de que la planificación estratégica formal es uno de esos impulsores, ya que crea conciencia y formula respuestas a las demandas de las partes interesadas para la RSC. Sin embargo, explorar relaciones de variables individuales es problemático, ya que otros factores endógenos importantes deben considerarse al explicar la RSC. Un esfuerzo formal de planificación estratégica está positivamente relacionado con la RSC. Además, después de considerar los esfuerzos formales de planificación de una empresa (Galbreath, 2010).

El proceso de globalización ha alterado significativamente la naturaleza, el comportamiento y el poder de las corporaciones, así como las relaciones entre las empresas y la sociedad, los gobiernos y otras partes interesadas. En las últimas décadas, los estados nacionales han experimentado un debilitamiento de su soberanía en términos de su capacidad para ajustar sus políticas económicas nacionales. Esto se debe en parte a la institucionalización internacional y a una cesión voluntaria de competencias nacionales a entidades supranacionales como la Unión 
Europea (UE) o la Organización Mundial del Comercio (OMC). Pero también las empresas pudieron influir en la legislación nacional. El aumento de la movilidad y los menores costos de comunicación y transporte permitieron a las multinacionales trasladar sus lugares de negocios al país ofreciendo el marco legal más atractivo y los costos laborales más bajos. En los mercados abiertos, las empresas (incluso las más pequeñas sin un lobby influyente) pueden elegir entre varias alternativas y, por lo tanto, están menos sujetas a la legislación nacional. El artículo se centrará en el desarrollo de un marco metodológico para la evaluación de las acciones de RSC a lo largo de todo el proceso de planificación estratégica, esencial para el éxito de los programas de RSC. Para que la RSC se integre en la estrategia comercial se requiere repensar la misión, la visión y la finalización de las estrategias de la organización en el espíritu de la RSC. Un marco metodológico coherente y fácil de aplicar facilitará la integración de la RSC que podría conducir al logro de cualquier objetivo fundamental de las estrategias organizacionales, que es la armonización de los dos objetivos fundamentales de una organización contemporánea: maximizar los beneficios y satisfacer las necesidades y requisitos de todos los interesados.

\section{1. Ética en la Planeación estratégica}

De acuerdo con Wilhelm (2010) en la discusión sobre ética empresarial, dos conceptos parecen ser irreconciliables y fundamentalmente conflictivos: moral y lucro (ganancias). Los empleadores o accionistas pueden afirmar que el aumento de la competencia a menudo obliga a las empresas a tomar medidas impopulares para reducir los costos a fin de seguir siendo rentables. Los críticos de la globalización, los activistas ambientales o los portavoces de los sindicatos, por el contrario, argumentan que las empresas abusan de su libertad y de una legislación demasiado liberal (Galbreath, 2009). Que se simpatice más con la primera o la segunda posición, es un hecho que los líderes empresariales con frecuencia enfrentan situaciones de dilema y deben tomar una decisión entre una alternativa (más bien) moralmente legítima o (más bien) que aumenta las ganancias que debe comunicarse y explicado a las partes interesadas y accionistas respectivamente (Chacón \& Rugel, 2018).

El economista liberal Milton Friedman intentó resolver esta situación localizando las responsabilidades corporativas únicamente en el campo económico: La responsabilidad social de las empresas es aumentar sus ganancias (Husted \& Allen, 2006). Esta declaración claramente tenía un propósito provocador, pero también considera el marco institucional y los principios básicos de un sistema capitalista (economía de mercado, estructuras de incentivos, competencia, derechos de propiedad, etc.) lógicamente desde una perspectiva liberal y agrega ideas valiosas al debate de la RSC. De acuerdo con Ramos et al.(2017) la argumentación de Friedman es doble:

Primero, niega que una corporación misma pueda tener responsabilidades y, por lo tanto, ve obligaciones morales solo a nivel individual. Por lo tanto, los gerentes tienen principalmente un deber fiduciario con los propietarios de la empresa, que generalmente será ganar la mayor cantidad de dinero posible. El individuo puede sentirse responsable de muchos asuntos diferentes, como los derechos de las minorías, la protección del medio ambiente, la caridad, su vecindario, los refugios para desamparados, los clubes deportivos, la educación de niños desfavorecidos, etc. y es muy libre de dedicar sus ingresos personales, tiempo y esfuerzo a estas causas, pero no los recursos que le confían los propietarios de la empresa. Tiene que haber una diferenciación distintiva entre el ejecutivo o el agente designado para dirigir una corporación y la persona privada que luego actúa como director.

Segundo, Friedman implica que la obligación social de una empresa es mantenerse rentable y en el negocio. Al emplear y pagar trabajadores, producir productos y ofrecer servicios a las necesidades de la sociedad, invertir en nuevas tecnologías y crear empleos, una empresa ya aumenta decisivamente la prosperidad general. En otras palabras, sería poco ético e irresponsable de una empresa arriesgar su existencia y los trabajos de los empleados al reducir su competitividad a través del compromiso social. Además, la obtención de ganancias es un indicador confiable del desempeño del ejecutivo, mientras que es difícil evaluar en qué medida se resolvió un problema 
social o ecológico mediante la disposición de la equidad de los accionistas (dado el caso de que incluso los propietarios lo deseaban).

Friedman opina que la responsabilidad social más bien pertenece a los políticos y funcionarios estatales que a los gerentes corporativos. Es tarea del estado deducir impuestos de los ingresos de empleadores y empleados y gastarlos de tal manera que sirvan de manera óptima al interés público (redistribución de ingresos, mejora del sistema educativo, financiamiento cultural, proyectos de forestación, calificación) programas para desempleados permanentes, etc.) (Vergara et al., 2020). Además, es deber del gobierno establecer el marco legal para las empresas a fin de perseguir motivos sociales o ecológicos (por ejemplo, protección contra despidos o límites máximos para las tasas de emisión) (Román \& Pérez, 2018). En general, todas las empresas enfrentan las mismas restricciones legales y las mismas condiciones de mercado, mientras que una compañía que incurre en significativamente más gastos en el campo de las responsabilidades sociales que otras corre el riesgo de ser menos competitiva en precio que sus competidores. Una empresa que invierte capital y esfuerzo únicamente en proyectos sociales en lugar de nuevas innovaciones podría verse expulsada del negocio por el proceso de destrucción creativa a la larga.

Friedman observó que la RSC podría en última instancia enfrentar los objetivos corporativos con los objetivos sociales (Sitnikov, 2017). Hay una única responsabilidad social de las empresas: usar sus recursos y participar en actividades diseñadas para aumentar sus ganancias siempre que se mantenga dentro de las reglas del juego, es decir, se involucra en una competencia abierta y libre sin engaños o fraude (Hamid, 2010). En la opinión de Moore (2020) la RSC crea impedimentos en el funcionamiento de los negocios y puede generar confusión sobre los verdaderos objetivos de la empresa, con el crecimiento en la complejidad de los negocios y las preocupaciones sobre la sostenibilidad, puede haber un conflicto entre la mejora de la rentabilidad a largo plazo de una empresa y su contribución al bien público. La situación a menudo se ve exacerbada por la aparente falta de recompensas al seguir una estrategia de RSC (Dagnino, 2008).

\subsection{Responsabilidad Social Corporativa}

Rojas et al. (2020) mencionan que, si bien son importantes para el administrador las fases de la planeación estratégica, también hay que destacar las actividades de RSC para identificarlas y considerarlas en la planificación, mismas que han pasado de ser predominantemente filantrópicas a tener un enfoque de valores más compartidos. Hoy en día, las empresas exitosas se centran en los problemas socialmente responsables que son directamente relevantes para sus operaciones. Las actividades de RSC se dividen en cuatro categorías principales. No son mutuamente excluyentes, así que habrá que definir formas de incluirlos en la propia e individual (desde el punto de vista de la empresa) planificación corporativa y comercial.

1) La responsabilidad filantrópica implica retribuir a la comunidad y donar a causas dignas en forma de tiempo, dinero u otros recursos.

2) La responsabilidad ambiental implica esfuerzos para reducir su huella de carbono mediante la adopción de políticas amigables con el medio ambiente.

3) La responsabilidad ética requiere que la empresa y todas las personas de su cadena de suministro utilicen prácticas comerciales justas. Esto afecta la forma en que se trata a los empleados, se ganan contratos y se realizan negocios.

4) La responsabilidad económica se refiere a operar con prácticas comerciales filantrópicas, ambientales y éticas sólidas de manera rentable y que pueda sostener el crecimiento a largo plazo.

\subsection{Desempeños de responsabilidad social}


El desempeño de responsabilidad ambiental es el que representa la capacidad de las empresas manufactureras para reducir los impactos ambientales a través de la reducción de las emisiones de carbono, la gestión de residuos y el diseño automático de productos para controlar el riesgo de peligros ambientales y el efecto del cambio climático. En sostenibilidad, la sostenibilidad ambiental de campo es la principal prioridad de la gestión. La responsabilidad ambiental y el rendimiento aumentan la productividad, así como la visibilidad del mercado de las empresas manufactureras ante diversos interesados. Este estudio considera preguntas que explican las iniciativas de gestión para controlar el riesgo de accidentes ambientales, la reducción de riesgos físicos y la mejora tecnológica en el proceso de producción que permiten garantizar el desempeño ambiental (Farfán, 2018). El desempeño de responsabilidad económica explica la capacidad de una empresa para maximizar la rentabilidad reduciendo costos y mejorando la calidad de vida de los empleados. El desempeño económico alienta a la gerencia corporativa a tomar iniciativas sostenibles. Este estudio considera las preguntas específicas sobre la base de diversas protecciones y oportunidades de los interesados, calidad de vida de la fuerza laboral, oportunidades de empleo y desarrollo económico del país. Todas las preguntas están diseñadas para explicar la percepción de los empleados sobre el progreso de sostenibilidad económica de la corporación (Guerrero et al., 2017).

El desempeño de Responsabilidad Social representa el desempeño organizacional con respecto a la legitimidad social que asegura un fuerte vínculo entre las empresas y la sociedad. Las organizaciones están haciendo negocios en la sociedad y, como resultado, tienen la gran responsabilidad de cuidar a los actores sociales. Por lo tanto, la organización debe emprender buenas iniciativas para garantizar un lugar de trabajo saludable, una política salarial estándar, la seguridad y la libertad de expresión de los trabajadores. Al garantizar oportunidades y beneficios a los trabajadores internos, reconoce ampliamente la responsabilidad ante toda la sociedad (Sarabia et al., 2019). El desempeño de Responsabilidad Social Corporativa declara la contribución de la organización a las diferentes partes interesadas de la sociedad, incluidos empleados, clientes, inversores, personas de la comunidad, gobierno, autoridades locales, medios de comunicación, sociedad civil y activistas ambientales. Crea valores para la organización, tanto interna como externamente. Kanji y Chopra (2010) definen a la RSC como un componente integral de las operaciones de una empresa mediante el cual contribuye voluntariamente a la sociedad en términos de inversión económica, ambiental, ética y social. La planificación estratégica organizacional y las políticas y estrategias a corto y largo plazo, así como las actividades de RSC, están diseñadas para mejorar el desempeño que es una herramienta importante que permite a la organización superar todos los inconvenientes posibles al considerar los programas y funciones apropiadas.

\subsection{Relación entre la PE y RSC}

En la larga historia de los negocios, la RSC es un concepto relativamente nuevo. Durante mucho tiempo, las empresas creyeron que sus únicas responsabilidades eran ganar la mayor cantidad de dinero posible y maximizar el valor para sus accionistas (Kriyantono, 2015). La planificación estratégica y la responsabilidad social corporativa es una forma de gestión en la que las empresas toman en consideración los aspectos éticos de sus operaciones comerciales. Incorporan estas preocupaciones sociales en sus estrategias comerciales y son más conscientes de sus roles en la sociedad y sus comunidades fuera de los negocios. Más que simplemente obedecer la ley, la responsabilidad social corporativa implica que una empresa tome medidas proactivas para mejorar la calidad de vida de sus empleados y la comunidad (Heikkurinen, 2018). Diferentes empresas seleccionarán una estrategia de responsabilidad social diferente entre sí, pero todas se centran en cuatro aspectos éticos de los negocios: económico, ético, legal y filantrópico.

La RSC impacta significativamente en el desarrollo e implementación de liderazgo y gestión estratégicos. Los líderes deben realizar cambios básicos para garantizar que se establezcan las prioridades para la RSC, se construya la confianza en la organización y se alcancen los objetivos establecidos. Los gerentes pueden 
desarrollar nuevos Códigos de conducta que, cuando se refuerzan mediante la capacitación, un liderazgo sólido y se alienta a través de varios métodos de desempeño, pueden establecer un alto estándar de comportamiento corporativo (Carranco et al., 2014). Los gerentes y las organizaciones deben asegurarse de que todas las políticas y sistemas que fomentan la RSC más allá de los requisitos legales estén alineados con los valores estratégicos, la visión y los objetivos generales de la empresa en su conjunto (Moore, 2020; Rojas et al., 2020; Vergara et al., 2020). Según Landor Associates (2020), el $77 \%$ de los consumidores dice que es importante que las empresas sean socialmente responsables. En la primera década del siglo XXI, solo una docena de compañías Fortune 500 emitieron un informe de Responsabilidad Social Corporativa (RSC). Ahora, la mayoría lo hace, y más de 7,000 empresas en todo el mundo han firmado el Pacto Mundial de la Organización de Naciones Unidas (ONU) comprometiéndose a mostrar una buena ciudadanía global. Además, muchas empresas están siguiendo el ejemplo de expertos en estrategia, como Michael Porter, e integran la RSC en su estrategia y operaciones organizativas.

Figura 1

Enfoque del plan estratégico e integrado a RSC

\begin{tabular}{|c|c|c|}
\hline $\begin{array}{c}\text { Impactos sociales } \\
\text { generales }\end{array}$ & $\begin{array}{l}\text { Impactos sociales de la } \\
\text { cadena de valor }\end{array}$ & $\begin{array}{l}\text { Dimensiones sociales del } \\
\text { contexto competitivo }\end{array}$ \\
\hline Buen ciudadano & $\begin{array}{c}\text { Mitigar el daño de las } \\
\text { actividades de la cadena de } \\
\text { valor }\end{array}$ & $\begin{array}{l}\text { Capacidades estratégicas de } \\
\text { apalancamiento de filantropía para } \\
\text { mejorar áreas destacadas de } \\
\text { contexto competitivo }\end{array}$ \\
\hline RSC receptiva & $\begin{array}{c}\text { Transformar las actividades de } \\
\text { la cadena de valor para } \\
\text { beneficiar a la sociedad al } \\
\text { tiempo que refuerza la } \\
\text { estrategia. }\end{array}$ & \\
\hline \multicolumn{3}{|c|}{ EVALUACIÓN DE LA CARTERA ACTUAL } \\
\hline Débil & & Fuerte \\
\hline
\end{tabular}

La figura 1 ilustra un enfoque publicado en Harvard Business Review en 2006 basado en construir un plan estratégico e integrado de RSC mejorará el impacto en la comunidad, aumentará el reconocimiento por la ciudadanía corporativa excepcional y el servicio comunitario, e impactar el rendimiento a través de una mayor participación de los empleados y la comunidad. Se está haciendo más común entre las empresas establecer un común filantrópico, para al inmiscuir a la planeación estratégica en la RSC se deberá tener una razón financiera que medir la misma se le llama como retorno de la inversión social (SROI).

Otro elemento importante de la planificación estratégica actual es la responsabilidad social corporativa (RSC), donde los gerentes enfrentan una demanda variada y creciente de los interesados. Esta demanda ha estado marcada por numerosas afirmaciones que vinculan la responsabilidad social corporativa con las ganancias de una empresa, particularmente en publicaciones profesionales, en los medios de comunicación y por organizaciones internacionales emergentes de RSE, como el Pacto Mundial de las Naciones Unidas (Moura-Leite \& Padgett, 2014). La motivación es loable, si se puede demostrar una relación RSE positiva - desempeño financiero, y luego las empresas estarán motivadas para aumentar el gasto en actividades de RSE (Flores \& Cano, 
2016). Sin embargo, la investigación académica hasta la fecha no ha sido concluyente, ya que ha encontrado relaciones positivas, negativas y curvilíneas entre la RSE y el desempeño financiero. Por lo tanto, la RSE se enfoca en dos áreas: los comportamientos internos, que se refieren a la forma en que una corporación lleva a cabo las operaciones diarias de sus funciones comerciales centrales, y los comportamientos externos, que se refiere al compromiso de una corporación fuera de su interés comercial. Va más allá de las buenas tácticas de relaciones públicas o de ser agradable. La planificación del comportamiento interno generalmente comienza en el Departamento de Recursos Humanos. Puede ser una ayuda para el reclutamiento y la retención. Los ejemplos son "ir verde", igualar las contribuciones caritativas de los empleados; creando programas de 'ayuda a la comunidad' y patrocinando eventos comunitarios (Sitnikov, 2017).

Si bien los comportamientos externos pueden incluir los últimos tres comportamientos internos, difieren de la RSE interna porque la administración y las relaciones públicas considerarán el impacto financiero de sus decisiones debido a sus partes interesadas, como los propietarios y accionistas. El negocio existe por muchas razones, pero la supervivencia depende en última instancia de las ganancias(Guerrero et al., 2017). Las corporaciones crean impactos sociales y sociales, tanto positivos como negativos a través de las operaciones diarias de su cadena de valor. Las corporaciones y las sociedades en las que operan ya están entrelazadas. Las sociedades necesitan corporaciones para dar a su gente empleo e infraestructura, y las corporaciones necesitan sociedades saludables para proporcionar una fuerza laboral capaz. Aunque la sociedad mira en muchos casos al mundo corporativo en lugar de al gobierno para la provisión de empleo e infraestructura (sin mencionar los bienes y servicios), es solo una sociedad saludable que puede crear el tipo de trabajadores productivos que toda corporación busca contratar (Ramos et al., 2017).

Las empresas existen para crear prosperidad. La sociedad, a su vez, decide qué límites imponer sobre el comportamiento de las empresas y, por lo tanto, tenemos leyes para proteger el bien común. La mayor parte del mundo ha alcanzado la etapa en la que existen buenas leyes, pero existe una aplicación deficiente de esas leyes. La mala aplicación tiene sus raíces en la corrupción y las instituciones débiles, y la mala gobernanza perpetúa la pobreza. Los programas de responsabilidad social corporativa intentan cerrar la brecha entre las leyes vigentes y las vigentes, y los fundamentos básicos de las buenas prácticas comerciales, como la obediencia a las leyes locales, la evitación de prácticas de explotación y la total transparencia.

\subsection{Retorno de la inversión social}

En esencia, SROI mide si los beneficios del trabajo superan sus costos. Al igual que su contraparte corporativa mundial (retorno de la inversión), SROI cuantifica y monetiza el valor económico y social que es probable que un programa cree y compara esos beneficios con los costos operativos del programa durante un período de años (Flockhart, 2005). Por ejemplo, un programa de preparación de la fuerza laboral que aumenta el potencial de un individuo para obtener un trabajo a tiempo completo puede reclamar beneficios sociales como un menor costo de asistencia gubernamental (subsidios de vivienda, cupones de alimentos, bonos de asistencia social) y un mayor flujo de efectivo (ingresos individuales, recaudación de impuestos). Los costos operativos asociados incluyen los costos programáticos y generales para brindar servicios de obtención de empleo (Ali et al., 2019).

Sin embargo, hay algunas cosas que SROI no mide. Si bien SROI es una forma de medir el impacto de un programa, no prueba la causalidad y no puede reemplazar la medición de los resultados reales. La fuerza de un modelo SROI aumenta a medida que la organización obtiene evidencia de sus resultados a través de la investigación de mejores prácticas o estudios de autoevaluación. También es importante tener en cuenta que este modelo no es integral, hay muchos beneficios sociales no cuantificables, como el aumento de la confianza en uno mismo o la estabilidad familiar (Klemelä, 2016). El SROI de un programa (también conocido como relación costo-beneficio) debe ser mayor que 1. Es decir, por cada $\$ 1$ invertido en el programa, se deben crear más de $\$$ 1 en beneficio social para justificar el gasto. Dicho de otra manera, el resultado final (o beneficio) del programa 
es su impacto. Los ciudadanos gastan miles de dólares en educación universitaria, esperando obtener más ingresos a través de trabajos mejor pagados de lo que cuesta obtener esa educación. Si el SROI del programa es inferior a 1, la intervención puede generar menos beneficios para los clientes y la sociedad de lo que cuesta operar. Cuando ese es el caso, la organización puede considerar actividades alternativas que puedan producir un mayor beneficio al mismo costo.

La fuerza de SROI radica en su capacidad para demostrar el impacto. Las organizaciones sin fines de lucro pueden usarlo como una poderosa herramienta de narración de historias para compartir su valor para la comunidad en llamamientos anuales y conectarse a formas de inversión de impacto, como bonos de impacto social o pagar por el éxito. Del mismo modo, los inversores sociales pueden usarlo para evaluar el éxito y el pago de inversiones sociales similares. Para comenzar a investigar este enfoque, Vluggen et al., (2020) recomiendan seguir los siguientes pasos:

- Trabajar con un director financiero y (o) presidente de finanzas para asegurarse de tener una idea clara de los costos relacionados con el programa, tanto directos como indirectos, utilizando una metodología como el costo basado en actividades

- Trabajar con un equipo de programa y (o) evaluadores para realizar una investigación secundaria sobre:

- Análisis de costo-beneficio o estimaciones de SROI de otras organizaciones con un modelo de programa similar.

- Evaluar los beneficios individuales y comunitarios generados por el modelo del programa (por ejemplo, menos dependencia de la asistencia del gobierno, mayor probabilidad de un trabajo mejor remunerado).

- Investigar herramientas SROI populares, como la desarrollada por REDF (2017), para decidir cómo construir el mejor modelo para una organización

- Reunir a los equipos de finanzas y programas para finalizar un modelo de alto nivel y comenzar a ingresar datos para determinar un cálculo de SROI único para la organización

- Hacer una prueba de presión del modelo con su junta y los financiadores locales para asegurarse de capturar con precisión su impacto

- Actualizar el modelo basado en un nuevo programa y datos financieros y nuevas investigaciones en su campo.

\section{Metodología}

El análisis documental es un procedimiento sistemático para revisar o evaluar documentos, tanto impresos como electrónicos (basados en computadora y transmitidos por Internet). Al igual que otros métodos analíticos en la investigación cualitativa, el análisis de documentos requiere que los datos serán examinados e interpretados, la comprensión de ganancia, y se desarrollan de forma empírica. El análisis de documentos a menudo se usa en combinación con otros métodos de investigación cualitativa como un medio de triangulación.

\section{Resultados}

Quizás lo más importante es esta opinión de que el verdadero impacto del trabajo colectivo que se lleva a cabo en el sector sin fines de lucro está subestimado por quienes están dentro y fuera del sector debido a la ausencia de métricas apropiadas por las cuales se pueda rastrear la creación de valor, calculado y atribuido a las inversiones filantrópicas y públicas que financian esos impactos. En el sector con fines de lucro, se habla de relaciones precio / ganancias y rendimiento del fondo de cartera. De hecho, al cierre de cada día se sabe exactamente qué rentabilidad financiera ha generado el mercado. Por el contrario, las organizaciones sin fines de lucro no tienen métricas equivalentes para reclamar el valor creado a través de su trabajo. Esta falta de 
métricas transferibles subyace a una serie de problemas que enfrenta el sector, que van desde dificultades en la recaudación de fondos hasta la incapacidad de proporcionar al personal una compensación adecuada (Ali et al., 2019; Flockhart, 2005).

\subsection{Análisis SROI}

A medida que el sector sin fines de lucro continúa compitiendo por dineros caritativos limitados, se vuelve cada vez más importante que podamos comprender no solo que un programa es una buena causa, sino que sus beneficios sociales argumentan para aumentar las inversiones en el trabajo. Por lo tanto, se debe entender la creación de valor generado por empresas sociales y la documentación de esa creación de valor a través de la aplicación del análisis SROI (Vluggen et al., 2020).

Según Vluggen et al. (2020) un análisis SROI hace lo siguiente:

- Examinar una actividad de servicio social durante un período de tiempo determinado (generalmente de cinco a diez años).

- Calcular la cantidad de "inversión" requerida para respaldar esa actividad y analiza la estructura de capital de la organización sin fines de lucro que está implementada para respaldar esa actividad.

- Identificar los diversos ahorros de costos, reducciones de gastos y beneficios relacionados que se acumulan como resultado de esa actividad de servicio social.

- Monetizar esos ahorros de costos y beneficios relacionados (es decir, calcula el valor económico de esos costos en términos de dólares reales).

- Descontar esos ahorros al comienzo del plazo de inversión (denominado tiempo cero) utilizando un valor presente neto y (o) análisis de flujo de efectivo descontado

- Presenta el valor socioeconómico creado durante el período de tiempo de inversión, expresando ese valor en términos de valor presente neto y tasas y relaciones de retorno social de la inversión

El rendimiento puede medirse como una relación tal que el valor presente de los beneficios netos se divida por el valor presente de los costos totales o puede calcularse en función de un cálculo del rendimiento de la inversión utilizando una tasa de descuento o rango de tasas acordado (Bellucci et al., 2019).

Los beneficios netos de una inversión en una empresa social se componen de dos flujos de efectivo. El primer flujo de caja se genera a partir de las operaciones de la propia empresa social. Los flujos de efectivo comerciales se pronostican a diez años y a perpetuidad y luego se descuentan a una cifra de valor presente. El segundo flujo de efectivo es un cálculo del ahorro neto total para la sociedad, es decir, el valor económico de los impactos sociales del programa. Para los propósitos de explicación de este documento, el término sociedad se refiere específicamente a aquellas entidades gubernamentales sobre las cuales recae el costo social de la pobreza (Ali et al., 2019).

Crear valor social y socioeconómico es claramente beneficioso para los participantes individuales del programa y las comunidades, y también reconocemos que la carga inmediata de la pobreza recae sobre las familias y las comunidades. Sin embargo, los ahorros netos para la sociedad se componen de los impuestos adicionales generados por las operaciones de la empresa y la reducción de los costos de desempleo, los nuevos salarios de los empleados y los dólares adicionales que las empresas utilizaron asociados con su misión social, menos cualquier subvención e inversión filantrópica (Rojas et al., 2020).

Los salarios y los dólares adicionales utilizados para la misión social de las empresas, mientras que los costos para las empresas, se consideran beneficios para los empleados. Este flujo de efectivo se pronostica a diez años y a perpetuidad y luego se descuenta a una cifra de valor presente utilizando un rango de tasas de descuento. Los nuevos dólares de impuestos, ahorros netos y flujos de efectivo comerciales se descuentan utilizando las tasas 
de descuento apropiadas y luego se suman para formar los beneficios totales para la sociedad (Pathak \& Dattani, 2014).

El valor presente neto de los beneficios se divide por los costos totales de la organización. Los costos totales representan los dólares filantrópicos invertidos durante un año determinado u otro marco de tiempo de inversión. Esta cifra final representa una de las medidas de desempeño de la organización: su relación SROI. Otra medida de rendimiento es la tasa SROI, que se calcula realizando cálculos de la Tasa interna de rendimiento (TIR) basados en el valor socioeconómico total y los costos totales.

$$
\text { SROI }=\frac{\text { Valor presente de los beneficios }}{\text { Valor presente de los costos }}=\text { Ratio del retorno social }
$$

\section{Conclusiones}

En una era de redes sociales y noticias instantáneas, la reputación puede verse irreparablemente dañada en segundos. Pero la RSC no se trata solo de la gestión de riesgos y de desviar el escrutinio negativo. Hay un enorme valor en hacer el bien, especialmente cuando le permite diferenciarse.

- Imagen de marca más sólida: operar de manera responsable gana puntos, gana reconocimiento y solidifica su reputación. Centrarse en los problemas de RSC correctos puede contribuir hasta la cuarta parte del valor de la marca de la empresa.

- Aumento de la lealtad y las ventas de los clientes: los consumidores conscientes de hoy en día tienen relaciones más personales con los bienes y servicios que compran, buscando empresas que sean responsables y apoyen causas valiosas.

- Ahorro de costos operativos: disminuir su impacto ambiental a menudo conduce a formas innovadoras de reducir sus costos operativos, lo que mejora sus resultados.

- Reclutamiento y retención de empleados: los empleados se comprometen más y tienen contratos más largos con empresas que mantienen buenas prácticas de RSC. Según EDC (2020) un estudio reciente de Deloitte encontró que cuando se preguntó a los millennial sobre el propósito principal de los negocios, el $63 \%$ más eligió mejorar la sociedad en lugar de generar ganancias.

- Acceso a financiación: su reputación corporativa puede afectar su capacidad de obtener capital de riesgo, seguros, préstamos y financiación.

- Mejora las relaciones con los organismos reguladores: la mayoría de las empresas están reguladas de alguna manera, por lo que tener un buen nombre puede ayudar a aliviar y reducir la carga administrativa de su empresa.

- Operando claramente con políticas que valoran la diversidad entre la fuerza laboral.

- Otros esquemas pueden incluir trabajar con la comunidad local u organizaciones benéficas para crear actividades o empleos en el área

- Invertir en la educación y el desarrollo personal de los empleados.

- Las actividades de RSC deben integrarse en toda la organización y no centralizarse dentro de un departamento específico.

\section{Referencias bibliográficas}

Ali, M., Qureshi, M. I., \& Mustapha, I. (2019). SROI in Social Enterprises: A Systematic Literature Review of Recent Trends and Future Agenda. En L.-P. Dana \& V. Ratten (Eds.), Societal Entrepreneurship and Competitiveness (pp. 203-226). Emerald Publishing Limited. https://doi.org/10.1108/978-1-83867-471720191014 
Bellucci, M., Nitti, C., Franchi, S., Testi, E., \& Bagnoli, L. (2019). Accounting for social return on investment (SROI): The costs and benefits of family-centred care by the Ronald McDonald House Charities. Social Enterprise Journal, 15(1), 46-75. https://doi.org/10.1108/SEJ-05-2018-0044

Carranco, M. F. O., Verdesoto, G. P. D., \& Andino, B. F. A. (2014). La responsabilidad social corporativa en economías en desarrollo: Una visión de la realidad ecuatoriana. Revista ESPACIOS / Vol. 35 (N²) Año 2014. https://www.revistaespacios.com/a14v35n02/14350206.html

Chacón, J., \& Rugel, S. (2018). Responsabilidad social corporativa y su impacto positivo en la empresa y la sociedad. 12.

Dagnino, R. (2008). Responsabilidad Social Empresarial y Tecnología. https://www.revistaespacios.com/a09v30n01/09300151.html

EDC. (2020). 7 tips to build your own CSR strategy. https://www.edc.ca/en/article/7-tips-build-a-csrstrategy.html

Farfan, O. G. (2018). The financial system, its social responsibility, ethical conflicts and macroeconomic stability in Ecuador. Revista ESPACIOS, 39(32). https://www.revistaespacios.com/a18v39n32/18393227.html

Flockhart, A. (2005). Raising the profile of social enterprises: The use of social return on investment (SROI) and investment ready tools (IRT) to bridge the financial credibility gap. Social Enterprise Journal, 1(1), 29-42. https://doi.org/10.1108/17508610580000705

Flores, M., \& Cano, A. M. (2016). La responsabilidad social del profesional de las ciencias económicas, administrativas y contables como solución para la transparencia empresarial. REVISTA ESPACIOS, 37(36), 16373601-16373635.

Galbreath, J. (2009). Building corporate social responsibility into strategy. European Business Review, 21(2), 109-127. https://doi.org/10.1108/09555340910940123

Galbreath, J. (2010). Drivers of Corporate Social Responsibility: The Role of Formal Strategic Planning and Firm Culture. British Journal of Management, 21(2), 511-525. https://doi.org/10.1111/j.14678551.2009.00633.x

Guerrero, N. F., Parra, D. A., Rivera, J. L., Palma, P. E., \& Scrich, A. J. (2017). Caracterización y perspectivas de la Responsabilidad Social Empresarial en el contexto ecuatoriano. Revista ESPACIOS, 38(41). http://www.revistaespacios.com/a17v38n41/17384101.html

Hamid, K. T. (2010). STRATEGIC PLANNING (SP): A Corporate Social Responsibility Approach. https://www.academia.edu/1920665/STRATEGIC_PLANNING_SP_A_Corporate_Social_Responsibility_App roach

Heikkurinen, P. (2018). Strategic corporate responsibility: A theory review and synthesis. Journal of Global Responsibility, 9(4), 388-414. https://doi.org/10.1108/JGR-06-2018-0020

Husted, B., \& Allen, D. (2006). Strategic Corporate Social Responsibility and Value Creation among Large Firms. https://core.ac.uk/download/pdf/9426393.pdf

Kanji, G. K., \& Chopra, P. K. (2010). Corporate social responsibility in a global economy. Total Quality Management \& Business Excellence, 21(2), 119-143. https://doi.org/10.1080/14783360903549808 
Klemelä, J. (2016). Licence to operate: Social Return on Investment as a multidimensional discursive means of legitimating organisational action. Social Enterprise Journal, 12(3), 387-408. https://doi.org/10.1108/SEJ02-2015-0004

Kriyantono, R. (2015). Public Relations and Corporate Social Responsibility in Mandatory Approach Era in Indonesia. Procedia - Social and Behavioral Sciences, 211, 320-327.

https://doi.org/10.1016/j.sbspro.2015.11.041

Landor Associates. (2020). Planificación estratégica sin fines de lucro. https://socialimpactarchitects.com/services/corporate-social-responsibility/

Masud, Md. A. K., Rashid, Md. H. U., Khan, T., Bae, S. M., \& Kim, J. D. (2019). Organizational Strategy and Corporate Social Responsibility: The Mediating Effect of Triple Bottom Line. International Journal of Environmental Research and Public Health, 16(22). https://doi.org/10.3390/ijerph16224559

Moore, D. (2020, enero 23). Why Corporate Social Responsibility is Essential for Success. The Northridge Group. https://www.northridgegroup.com/blog/why-corporate-social-responsibility-is-essential-for-success/

Moura-Leite, R. C., \& Padgett, R. C. (2014). La evolución de la responsabilidad social de la empresa: Un abordaje histórico. Revista ESPACIOS / Vol. 35 (No 5) Año 2014.

https://www.revistaespacios.com/a14v35n05/14350409.html

Pathak, P., \& Dattani, P. (2014). Social return on investment: Three technical challenges. Social Enterprise Journal, 10(2), 91-104. https://doi.org/10.1108/SEJ-06-2012-0019

Ramos, E. L., Audet, X. L., \& Barrena-Martínez, J. (2017). Universities as Corporate Entities: The Role of Social Responsibility in Their Strategic Management. Corporate Governance and Strategic Decision Making. https://doi.org/10.5772/intechopen.69931

REDF. (2017, enero 24). Overview of SROI Analysis. REDFworkshop. https://redfworkshop.org/learn/overviewof-sroi/

Robin, D. P., \& Reidenbach, R. E. (1988). Integrating Social Responsibility and Ethics into the Strategic Planning Process. Business \& Professional Ethics Journal, 7(3/4), 29-46. JSTOR.

Rojas, C. P., Hernandez, H. G., \& Niebles, W. A. (2020). La transformación de la RSE como contribución activa de la gestión empresarial. Revista ESPACIOS, 41(01). http://www.revistaespacios.com/a20v41n01/20410104.html

Román, E. Y., \& Pérez, E. O. (2018). Impacto de la responsabilidad social empresarial en el clima organizacional. 20.

Saltos, Mary, \& Muñoz Moreta, E. R. (2019). La planificación elemento fundamental de la gobernanza en las instituciones públicas del Ecuador, periodo 2007-2017.

http://www.dspace.uce.edu.ec/handle/25000/17310

Saltos, Miguel, \& Velazquez, R. (2019). Apuntes teóricos para la promoción de la responsabilidad social empresarial en Ecuador. Revista ESPACIOS, 40(43). https://www.revistaespacios.com/a19v40n43/19404304.html

Sarabia, M. Y., García, L. C., \& Bravo, O. M. (2019). Responsabilidad social y turismo comunitario en la provincia Santa Elena. Revista ESPACIOS, 40(30). http://www.revistaespacios.com/a19v40n30/19403002.html 
Sitnikov, C. (2017). Relationships Between Corporate Social Responsibility and Strategic Planning. springerprofessional.de. https://www.springerprofessional.de/en/relationships-between-corporate-socialresponsibility-and-strate/11020766

Sousa, J. M. de, Soares, L., Pasa, C., \& Farache, F. (2010). Strategic corporate social responsibility management for competitive advantage. BAR - Brazilian Administration Review, 7(3), 294-309. https://doi.org/10.1590/S1807-76922010000300006

Vergara, A., Olalla, A., Yturralde, J. M., \& Sorhegui, R. (2020). Responsabilidad social corporativa RSC y su impacto en el rendimiento económico de las principales Empresas en Ecuador. Revista ESPACIOS, 41(10). https://revistaespacios.com/a20v41n10/20411013.html

Vluggen, R., Kuijpers, R., Semeijn, J., \& Gelderman, C. J. (2020). Social return on investment in the public sector. Journal of Public Procurement, 20(3), 235-264. https://doi.org/10.1108/JOPP-06-2018-0023

Wilhelm, T. (2010). La importancia estratégica de la responsabilidad social corporativa [Business economics Bachelor, Otto-von-Guericke-University Magdeburg]. https://www.grin.com/document/181835

Esta obra está bajo una Licencia Creative Commons Attribución-NoCommercial 4.0 International

(cc) BY-NC 